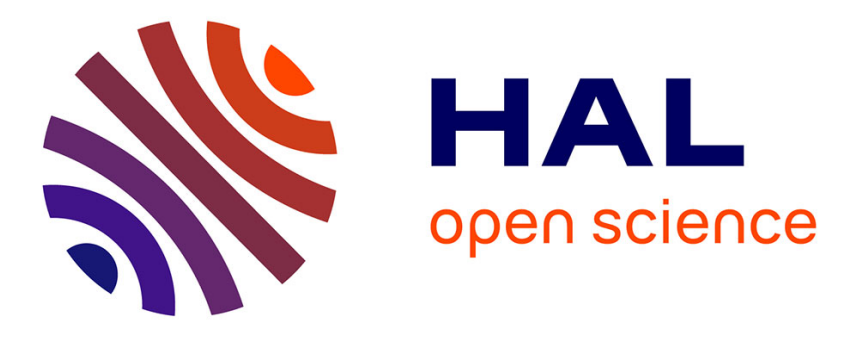

\title{
Maximizing the algebraic connectivity of meshed electrical pathways used as current return network
}

Etienne Goddet, Nicolas Retiere, Vojislav Stojanovic, Anca Dieudonne, Jerome Genoulaz, Jean-Michel Guichon

\section{- To cite this version:}

Etienne Goddet, Nicolas Retiere, Vojislav Stojanovic, Anca Dieudonne, Jerome Genoulaz, et al.. Maximizing the algebraic connectivity of meshed electrical pathways used as current return network. Mathematics and Computers in Simulation, 2019, 158, pp.18-31. 10.1016/j.matcom.2018.05.002 . hal-02350910

\section{HAL Id: hal-02350910 https://hal.science/hal-02350910}

Submitted on 30 Mar 2021

HAL is a multi-disciplinary open access archive for the deposit and dissemination of scientific research documents, whether they are published or not. The documents may come from teaching and research institutions in France or abroad, or from public or private research centers.
L'archive ouverte pluridisciplinaire HAL, est destinée au dépôt et à la diffusion de documents scientifiques de niveau recherche, publiés ou non, émanant des établissements d'enseignement et de recherche français ou étrangers, des laboratoires publics ou privés. 
archives-ouvertes

\section{Maximizing the algebraic connectivity of meshed electrical pathways used as current return network}

Etienne Goddet, Nicolas Retiere, Vojislav Stojanovic, Anca Dieudonne, Jerome Genoulaz, Jean-Michel Guichon

\section{To cite this version:}

Etienne Goddet, Nicolas Retiere, Vojislav Stojanovic, Anca Dieudonne, Jerome Genoulaz, et al.. Maximizing the algebraic connectivity of meshed electrical pathways used as current return network. Mathematics and Computers in Simulation, 2019, 158, pp.18-31. 10.1016/j.matcom.2018.05.002 . hal-02350910

\section{HAL Id: hal-02350910 \\ https://hal.archives-ouvertes.fr/hal-02350910}

Submitted on 30 Mar 2021

HAL is a multi-disciplinary open access archive for the deposit and dissemination of scientific research documents, whether they are published or not. The documents may come from teaching and research institutions in France or abroad, or from public or private research centers.
L'archive ouverte pluridisciplinaire HAL, est destinée au dépôt et à la diffusion de documents scientifiques de niveau recherche, publiés ou non, émanant des établissements d'enseignement et de recherche français ou étrangers, des laboratoires publics ou privés. 


\title{
Maximizing the algebraic connectivity of meshed electrical pathways used as current return network
}

\author{
Etienne Goddet ${ }^{\mathrm{a}, \mathrm{b}}$, Nicolas Retière ${ }^{\mathrm{a}, *}$, Vojislav Stojanović ${ }^{\mathrm{b}}$, Anca Dieudonné ${ }^{\mathrm{b}}$, \\ Jérôme Genoulaz ${ }^{\mathrm{b}}$, Jean-Michel Guichon ${ }^{\mathrm{a}}$ \\ ${ }^{\mathrm{a}}$ Univ. Grenoble Alpes, CNRS, Grenoble INP' , Grenoble, France \\ ${ }^{\mathrm{b}}$ Safran Electrical \& Power, 1 Rue Louis Blériot, 31702 Blagnac, France
}

\begin{abstract}
This paper proposes an innovative method for optimizing the connectivity of meshed electrical pathways used to carry return current and provide grounding in more composite aircrafts. In normal operation, these networks should ensure a low voltage drop between power sources and electrical loads. The networks are modelled as graphs and spectral graph analysis is used to study their complexity. Thanks to the insight into graph's spectrum, a multi-scale reading of meshed networks topology is proposed and an optimization problem is defined to decrease the DC resistance of the network while keeping constant its total weight. It is based on the maximization of the second eigenvalue of the graph's Laplacian matrix. The optimization problem is applied to a mock-up of a real aircraft current return network. The paper is concluded by checking the DC voltage drops in steady state conditions.
\end{abstract}

Keywords: Graph theory; Spectral analysis; Optimization; Algebraic connectivity; Current return networks

\section{Introduction}

In recent years, the conventional metallic materials used for aircrafts structure and fuselage have been replaced by new composite materials. This has been justified by the excellent mechanical properties of composites (e.g. their high resistance to fatigue and corrosion) and the reduction of aircraft weight they provide. The "standard" aircrafts benefit from metallic bodies which are commonly used as conductive pathways to carry the return of direct and alternating currents, faults currents, lightning currents and also to provide other functions related to grounding, electrostatic charge draining, electromagnetic shielding etc. Unfortunately, such benefits are not available on aircrafts made of

\footnotetext{
* Corresponding author.

E-mail address: nicolas.retiere@univ-grenoble-alpes.fr (N. Retière).

${ }^{1}$ Institute of Engineering Univ. Grenoble Alpes.
} 


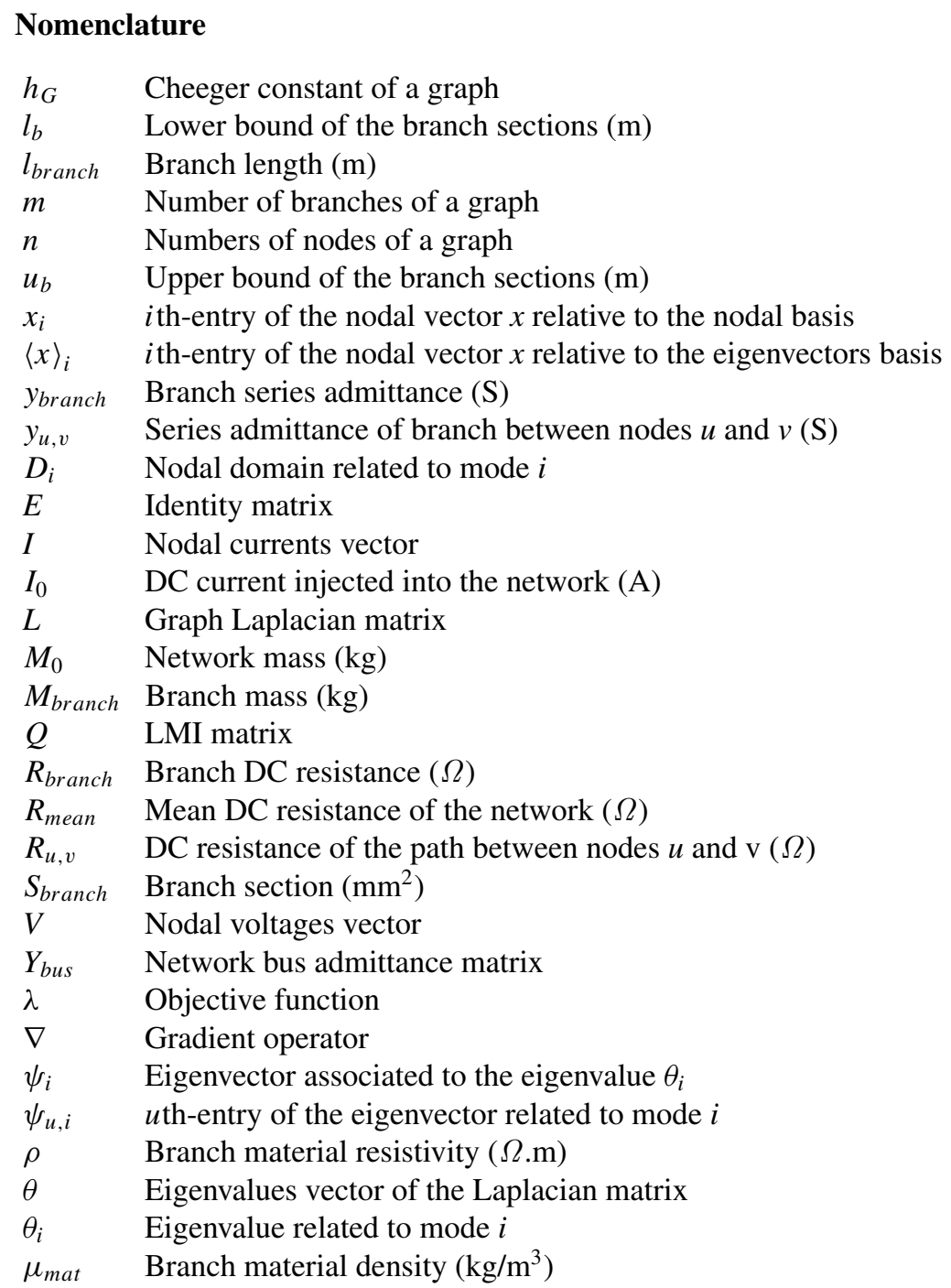

composite materials because of their low conductivity and bad thermal properties. Hence, composite aircrafts require an additional electrical network to provide the functions that the composite structures are not anymore able to ensure.

The additional electrical system can be a metallic network used to create a highly conductive electrical pathway for the return of currents. Such a network is practically realized by exploiting both structural metallic parts of the aircraft (beams, seats rails, etc.) and/or dedicated paths. Boeing has opted for a network made by the addition of long cables, bars and cable shielding for the current return network of the 787. Airbus, for its A350 airplane, has decided to reuse parts of the metallic structure of the aircraft to build a new Electrical Structure Network (ESN) [1,16,21]. This structure is composed of circular frames which support the composite structure, a cabin floor made by the assemblage of crossbeams and longitudinal elements (seat tracks, false tracks and raceways), a cargo floor constituted by crossbeams and roller tracks (longitudinal elements), several vertical elements which link the cabin floor to the cargo floor and the metallic cockpit (cf. Fig. 1).

Both Airbus and Boeing supplementary networks are meshed and embed many elements. This brings a lot of complexity in terms of construction. That is why a special attention has to be paid to the pre-sizing process. Predimensioning must ensure that the conditions for a safe operation of the aircraft electrical system are met while handling weight reduction. 


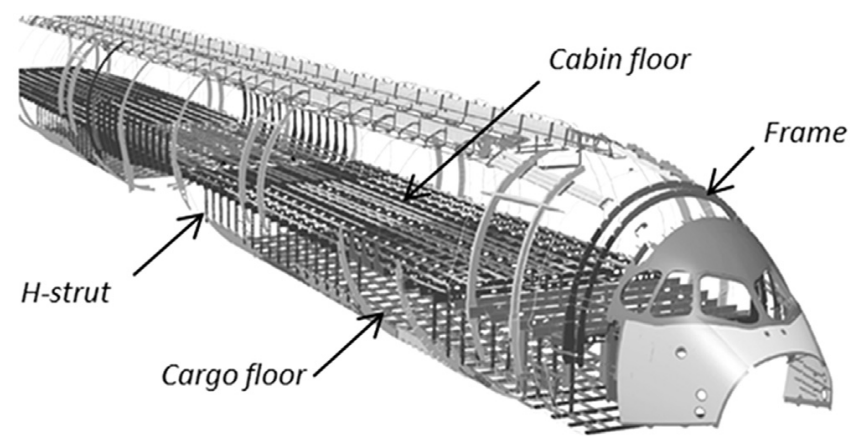

Fig. 1. ESN structure of A350 (picture taken from [1]).

This paper focuses on the direct current return function of the additional network. A good ground connection shall be made between every electrical loads and power sources. Actually, the additional network shall guarantee a voltage drop between loads and sources lower than $0.5 \mathrm{~V}$ in steady state conditions. A low impedance electrical pathway will facilitate the respect of this constraint. To decrease impedance, critical elements can be oversized. On the other hand, to cope with the objectives of fuel efficiency, the mass of the additional network must be sufficiently low to not cancel the advantages provided by the use of a composite structure. To find the best compromise between the weight reduction and the low impedance specification becomes therefore very challenging. This challenge is addressed in this paper.

To meet the low impedance and mass requirements, we present an optimization method to improve network connectivity of complex meshed networks. Our optimization strategy is based on the spectral theory of graphs instead of a circuit-oriented approach [17]. Spectral theory is already commonly used for complex graphs because it gives a multiscale reading of properties which cannot be visible by a simple visual inspection [19]. For instance, the complexity of meshed electrical circuits makes difficult for human brains to perceive which parameters drive the system properties (conductance, voltage drops, robustness, weight etc.) from the circuit-oriented representation. It is impossible to catch the most critical parameters without a long-last campaign of numerical simulations. On the other hand, the spectral graph theory enables to link the graph properties to the eigen modes, expressing the graph behaviour as a sum of modal behaviours. So, the spectral theory is expected to provide an easy reading of the properties of complex network and how they are linked with the topology and the design choices performed by engineers. Very few works based on spectral graph have been published in the field of power engineering. It is worth mentioning the use of spectral analysis to link eigenvalues and synchronizability of Central Italy HV power grid [14], the comprehensive investigation of energy transmission and synchronization by [24] or the works about graph spectra and dynamics in complex network of oscillators in [2] and [13].

Only DC operating conditions of current return networks will be investigated in this paper as more and more future aircraft electrical systems are designed to operate with DC systems. The underlying graph of the current return network will hence be described by its Laplacian matrix [2]. The optimization algorithm will lower network's DC resistance by maximizing the second eigenvalue of the Laplacian matrix [26].

The paper is organized as follows. Section 2 deals with the mathematical representation of electrical circuits by graphs. Section 3 outlines the spectral graph theory and the links with electrical and structural properties of networks. In Section 4, the definition of the optimization problem is explained. Section 5 presents the optimization of the mock-up of a meshed current return network called DEMLAB and developed by Safran Electrical \& Power.

\section{Graph representation of electrical networks}

In order to analyse the connectivity of a network and to provide some topological drivers for its design, a mathematical representation is required that will properly describe the interconnections between the network components. In this paper, the graph representation is adopted. It will be used to link the structural properties of the underlying graph of the network and its electrical properties such as DC conductance or voltage drops. Many different natural or human-engineered interconnected systems have already been studied using graph theory. In particular, 
power grids have been successfully investigated as complex graphs [23]. In our case, the graphs are undirected which means that all edges are bidirectional, since current flow is naturally bidirectional too. Moreover, graph edges are weighted by the corresponding branch series admittance.

The graph theory uses matrices to catch topological information of graphs. In this paper, the Laplacian matrix is used. Given a network with $n$ nodes and $m$ branches, the Laplacian matrix $L$ is a $(n \times n)$ matrix defined by:

$$
L_{u, v}=\left\{\begin{array}{c}
-y_{u, v} \text { if } u \text { and } v \text { are neighbours } \\
\sum_{t \in \text { nei }(u)} y_{u, t} \text { if } u=v \\
0 \text { otherwise. }
\end{array}\right.
$$

Two nodes are neighbours if they are connected by a branch and nei( $u$ ) is the set of neighbours of node $u . y_{u, t}$ is the series admittance of the branch between $u$ and $t$. Laplacian matrix is symmetric and the sum of its columns and rows are equal to zero. It is a singular matrix.

Electrical networks are often described by the primitive impedance matrix. It is a matrix whose elements are the self and mutual impedances of the network. If the network is only made of series impedances, the matrix elements referred by $(u, v)$ are equal to the admittance value $y_{u, v}$ connecting nodes $u$ and $v$. Of course, the matrix entry is equal to zero if there is no connection. The primitive impedance can give birth to new matrices that are more suitable for circuit-oriented analysis purposes. For instance, it is possible to build a bus admittance matrix $Y_{\text {bus }}$ that represents the electrical behaviour of the nodal variables of a meshed network such that [18]:

$$
Y_{\text {bus }} V=I
$$

$I$ and $V$ are the nodal current and voltage vectors. This matrix is used to perform electrical circuit calculations such as load-flows. It can be easily shown that the bus admittance matrix is also the Laplacian matrix of the underlying graph of the electrical network.

Some interesting properties of electrical networks can be caught by their graph-oriented mathematical representation. For instance, the node degree distribution of the underlying graph is often used as an indicator of performance $[4,11]$. Centrality measures based on betweenness properties are also useful to identify what are the central links and nodes $[5,22,25]$. Flow network problems allow to find the maximum current that can flow through an electrical grid while respecting thermal constraints of lines [15]. The total mass of a network can be estimated from the degree matrix. In this paper, the choice has been done to investigate the Laplacian matrix spectral properties, meaning the analysis of its eigenvalues and eigenvectors.

\section{Spectral analysis}

\subsection{Basic concepts}

Only the most relevant results for our study will now be presented. For a complete review of the spectral graph theory, the reader may refer to $[10,12]$.

The spectral graph theory is the study of the properties of the characteristic polynomials, eigenvalues and eigenvectors associated to the matrices describing the graph. For instance, the eigenvalues $\theta$ of the Laplacian matrix $L$ are the non-trivial roots of the characteristic equation:

$$
\operatorname{det}(L+\theta E)=0
$$

where $E$ is the identity matrix.

For a $(n \times n)$ matrix, solving Eq. (3) provides $n$ eigenvalues. Algebraic multiplicity of an eigenvalue is the multiplicity of its associated root. Since the Laplacian matrix is symmetric, semi-definite and positive, all its eigenvalues are positive real numbers. Furthermore, Laplacian matrix of any graph has at least an eigenvalue equal to zero because of its singularity. Algebraic multiplicity of the zero eigenvalue gives the number of connected components of the graph. By convention, eigenvalues are sorted in increasing order $\theta_{1}=0 \leq \theta_{2} \leq \cdots \leq \theta_{n}$. For a single connected component graph, the second smallest eigenvalue $\theta_{2}$ is called algebraic connectivity and the largest eigenvalue $\theta_{n}$ is called spectral radius. The algebraic connectivity is the core of our work because the higher it is, the better connected is the graph. Indeed, according to Cheeger's inequality, for two subgraphs 1 and 2 as shown in 


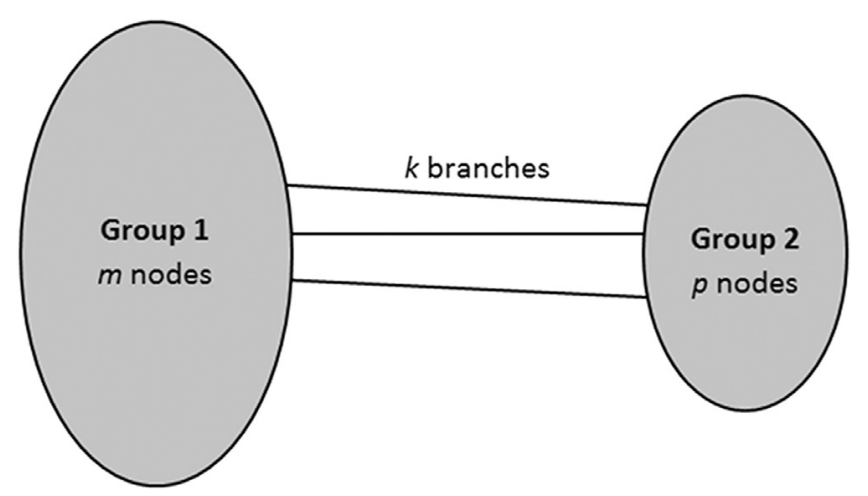

Fig. 2. Two subgraphs connected by a set of branches $k(m \geq p)$.

Fig. 2, high values of $\theta_{2}$ increase the minimal number of branches that are connecting the subgraphs [10]. To define the Cheeger's inequality, a parameter $h_{G}$ is introduced. It is the minimal ratio between the number of branches that have to be removed to divide the graph into two disjoint subgroups and the number of nodes of the smallest subgraph. The inequality of Cheeger is expressed by:

$$
\theta_{2} \leq 2 h_{G} .
$$

It means that the algebraic connectivity gives the inferior bound of the number of branches required to disconnect a subgroup of nodes from the main graph. Or, for a given number of removed branches, the algebraic connectivity gives the superior bound of the size of the smallest disconnected subgraph. A high algebraic connectivity should therefore correspond to a well-connected network.

To each eigenvalue $\theta_{i}$ is associated with an eigenvector $\psi_{i}$ defined by:

$$
L \psi_{i}=\theta_{i} \psi_{i} .
$$

For a real symmetric matrix, eigenvectors are orthogonal, meaning that $\psi_{i}^{t} \cdot \psi_{j}=0$ if $i \neq j$. And, every eigenvectors are normalized such that $\psi_{i}^{t} \cdot \psi_{i}=1$. All the components of the first eigenvector associated to the zero eigenvalue are therefore equal to $1 / \sqrt{n}$. All the other eigenvectors being orthogonal to the first eigenvector, it results in that the sum of their entries is equal to zero.

Each eigenvalue $\theta_{i}$ and its associated eigenvectors $\psi_{i}$ define the mode $i$.

\subsection{Spectral solving of the DC network equations}

A meshed DC electrical network is considered. It is supplied by constant current sources located at some nodes. The loads are modelled by constant current sources too. The network is balanced meaning that the total supply current is equal to the total load current.

The nodal currents vector $I$ is projected onto the orthogonal eigenvector basis such that:

$$
I=\sum_{i=1}^{n}\langle I\rangle_{i} \psi_{i}
$$

$n$ is the number of nodes. $\langle I\rangle_{i}$ is the $i$ th-component of the nodal currents vector relative to the eigenvectors basis. It is given by:

$$
\langle I\rangle_{i}=\psi_{i}^{t} . I .
$$

The modal components $\langle I\rangle_{i}$ of the current vector are an image of the contribution of each mode to the nodal excitation of the electrical system. It shall be noted that $\langle I\rangle_{1}$ is equal to zero as the network is balanced.

According to Eq. (2), the nodal voltages vector $V$ is given by:

$$
I=L V
$$


$L$ is the weighted Laplacian matrix of the graph related to the network. The weights are given by the conductance of the lines. The projection onto the eigen basis is therefore given by:

$$
\sum_{i=1}^{n}\langle I\rangle_{i} \psi_{i}=L \sum_{i=1}^{n}\langle V\rangle_{i} \psi_{i} .
$$

It results from the definition and orthogonality of the eigenvectors that for every $i$ except 1 :

$$
\langle V\rangle_{i}=\frac{\langle I\rangle_{i}}{\theta_{i}}
$$

$\langle V\rangle_{1}$ is undetermined because $\langle I\rangle_{1}$ and $\theta_{1}$ are both equal to zero. Its value depends on the choice of the reference voltage. For simplification purposes, we choose a zero value [24]. Hence, the nodal voltages vector is expressed as:

$$
V=\sum_{i=2}^{n} \frac{\langle I\rangle_{i}}{\theta_{i}} \psi_{i} .
$$

Considering Eqs. (6) and (11), the spectral analysis expresses the network's electrical behaviour as a sum of simple behaviours related to the modes.

\subsection{Voltage drops and nodal domains}

The voltage drops across the branches are defined as the gradient of the voltage vector, meaning the voltage difference between two neighbouring nodes. It is noted $\nabla V$ where the gradient $\nabla$ is defined by the transpose of the incidence matrix [9]. The voltage drops are therefore given by:

$$
\nabla V=\sum_{i=2}^{n} \frac{\langle I\rangle_{i}}{\theta_{i}} \nabla \psi_{i} .
$$

Voltage drops across the branches are linked to the nodal domains of the graph's eigenvectors.

A nodal domain $D_{i}$ related to mode $i$ is defined by [12]:

$$
\text { if } k, l \in D_{i} \text { then } \psi_{k, i} \psi_{l, i} \geq 0 \text {. }
$$

In this equation, $\psi_{k, i}$ (respectively $\psi_{l, i}$ ) is the $k$ th (resp. $l$ th) entry of the eigenvector $\psi_{i}$ associated with mode $i$. Every eigenvector component is attached to a node. For instance, $\psi_{k, i}$ is attached to node $k$.

The definition (13) means that two nodes $k$ and $l$ belong to the same domain $D_{i}$ if their related eigenvector components, respectively $\psi_{k, i}$ and $\psi_{l, i}$ are of the same sign. Some domains are therefore said to be positive if the signs of the eigenvectors components are positive. Negative domains are related to negative eigenvector entries. At the borders between two adjacent domains, the eigenvector components change of sign and the gradient of the eigenvector should be high.

According to the classical Courant's theorem, for every mode $i$, there is at most $i$ nodal domains [12]. A broad illustration is shown in Fig. 3 for an arbitrary 10-node graph. For the 2nd mode, there are only two domains, one positive and one negative. Indeed, the Courant's theorem states that there are at most two domains but the sum of the eigenvector components being equal to zero, some components must be positive where as some others must be negative. As a consequence, the exact number of domains for the second mode is exactly two. For the 10th mode, there are at most ten domains. The exact number of domains is not predictable by the theory. It could be less than ten. This strongly depends on the structure of the graph. Of course, if there are ten domains, every domain includes a single node and the sign of the eigenvector components change from node to node as it is broadly shown in Fig. 3.

The spectral graph theory provides therefore a multiscale reading of networks' connectivity. The second mode gives some indications about long-range connectivity of the system (i.e. connectivity between two sides of the graph, each side being covered by a nodal domain) whereas the higher modes are related to the local connectivity of nodes (i.e. connectivity between neighbours, each domain tending to be reduced to a few number of nodes).

Following Eq. (12), the critical voltage drops should be localized at the borders between nodal domains where the gradient is maximal due to the change of signs of the eigenvectors components. Because the voltage drops vary as the inverse of the eigenvalues, this effect should be magnified for the lower modes. 


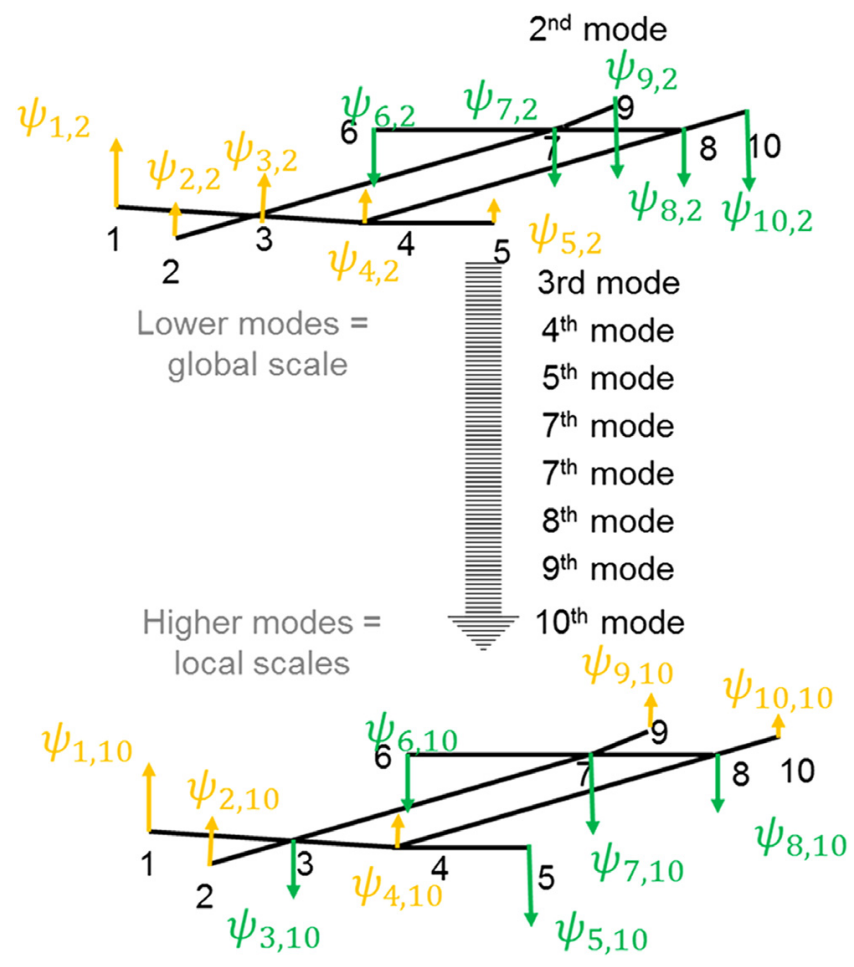

Fig. 3. Broad illustration of the nodal domains of a 10-node graph. Positive values of $\psi_{k, i}$ are shown by yellow ascending arrows. Negative values are shown by green descending arrows.

\subsection{Spectral decomposition of DC resistance}

Instead of looking at the voltage drops, the relation between modes and DC connectivity of the mesh grid can also be investigated through the spectral decomposition of the DC resistance of the path between two nodes. For that purpose, a constant nodal current is injected at node $s$ and withdrawn at node $l$. All the other nodal currents are set to zero. The nodal currents vector $I$ is therefore written as:

$$
I=\left(\begin{array}{c}
0 \\
\vdots \\
I_{l}=-I_{0} \\
\vdots \\
I_{S}=I_{0} \\
\vdots \\
0
\end{array}\right)
$$

The components of the decomposition of this vector onto the Eigen basis are given by:

$$
\langle I\rangle_{i}=\psi_{i}^{t} . I=\left(\psi_{s, i}-\psi_{l, i}\right) I_{0} .
$$

The voltage drop between nodes $s$ and $l$ is given by the difference of the nodal voltages. Considering Eqs. (11) and (15), it is expressed by:

$$
V_{s}-V_{l}=\sum_{i=2}^{n} \frac{\langle I\rangle_{i}}{\theta_{i}} \psi_{s, i}-\sum_{i=2}^{n} \frac{\langle I\rangle_{i}}{\theta_{i}} \psi_{l, i}=\sum_{i=2}^{n} \frac{1}{\theta_{i}}\left(\psi_{s, i}-\psi_{l, i}\right)^{2} I_{0} .
$$


The DC resistance is therefore given by:

$$
R_{s, l}=\frac{V_{s}-V_{l}}{I_{0}}=\sum_{i=2}^{n} \frac{\left(\psi_{s, i}-\psi_{l, i}\right)^{2}}{\theta_{i}} .
$$

As voltage drops, the DC resistance value is driven by the gradients of eigenvectors and by the inverse of eigenvalues. Because the eigenvalues are ranked in ascending order such that $\frac{1}{\theta_{n}} \leq \cdots \leq \frac{1}{\theta_{2}}$, DC resistance should be dominated by lower modes, especially by the algebraic connectivity $\theta_{2}$. Of course, it will depend on the nodes location too, meaning whether the two nodes are inside the same domain or not. This location will influence the values of eigenvectors gradients. For instance, if the nodes $s$ and $l$ are inside the same domain, it is expected that the DC resistance of the path is lower than if the nodes are in two distinct domains.

It results that DC connectivity of meshed electrical system is driven by its multiscale organization into nodal domains. An idea of its typical value is obtained by the average connectivity, expressed by the arithmetic mean of the DC resistance between two nodes. This mean resistance is defined by the sum of the individual path resistance divided by the number of pairs of distinct nodes $s$ and $l$ of the network.

$$
R_{\text {mean }}=\frac{1}{n(n-1)} \sum_{s=1}^{n} \sum_{l=1}^{n} R_{s, l} \text {. }
$$

Using the previous expression of $R_{s, l}(17)$ :

$$
R_{\text {mean }}=\frac{1}{n(n-1)} \sum_{s=1}^{n} \sum_{l=1}^{n} \sum_{i=2}^{n} \frac{\left(\psi_{s, i}-\psi_{l, i}\right)^{2}}{\theta_{i}}
$$

Developing $\left(\psi_{s, i}-\psi_{l, i}\right)^{2}$, the expression of the mean DC resistance becomes:

$$
R_{\text {mean }}=\frac{1}{n(n-1)} \sum_{i=2}^{n} \frac{1}{\theta_{i}}\left[\sum_{s=1}^{n}\left(\sum_{l=1}^{n} \psi_{s, i}^{2}+\sum_{l=1}^{n} \psi_{l, i}^{2}-2 \psi_{s, i} \sum_{l=1}^{n} \psi_{l, i}\right)\right] .
$$

Manipulating the summations over $s$ and $l$, it is possible to write:

$$
R_{\text {mean }}=\frac{1}{n(n-1)} \sum_{i=2}^{n} \frac{1}{\theta_{i}}\left[2 \sum_{s=1}^{n} \sum_{l=1}^{n} \psi_{l, i}^{2}-\sum_{s=1}^{n}\left(2 \psi_{s, i} \sum_{l=1}^{n} \psi_{l, i}\right)\right] .
$$

Due to the normalization of the eigenvectors, $\sum_{l=1}^{n} \psi_{l, i}^{2}=1$. Hence:

$$
R_{\text {mean }}=\frac{1}{n(n-1)} \sum_{i=2}^{n} \frac{1}{\theta_{i}}\left[2 \sum_{s=1}^{n} 1-\sum_{s=1}^{n}\left(2 \psi_{s, i} \sum_{l=1}^{n} \psi_{l, i}\right)\right] .
$$

For any $\mathrm{i} \geq 2, \sum_{l=1}^{n} \psi_{l, i}=0$ because all the eigenvectors are orthogonal to the unitary eigenvector $\psi_{1}$. Therefore:

$$
R_{\text {mean }}=\frac{1}{n(n-1)} \sum_{i=2}^{n} \frac{2 n}{\theta_{i}} .
$$

Since eigenvalues are sorted in an increasing order, it is obvious that, in average, the DC resistance of the network is limited by the inverse of the algebraic connectivity, i.e.:

$$
R_{\text {mean }} \leq \frac{2}{\theta_{2}} .
$$

Consequently, and independently of the nodal domains distribution, DC impedance of the meshed grid should be lowered in average by a higher algebraic connectivity. This has motived the formulation of an optimization problem based on the maximization of the second eigenvalue.

\section{Looking for an optimal network}

In order to increase the connectivity, and therefore to decrease the voltage drops across meshed networks used for carrying direct current, the optimization process is intended to maximize the algebraic connectivity for a given 
network's mass. Indeed, increasing performances of on-board electrical systems shall not be paid by an extra-mass of the overall structure. The geometry of the network will not be changed by the optimization process, meaning that the nodes will not be moved of location. Only the branch sections will be changed. However, their variations will be kept bounded. In this section, two optimization formulations are proposed and tested.

\subsection{Explicit maximization}

Branch weights are given by branch admittances $y_{\text {branch }}$. They are defined as follows:

$$
y_{\text {branch }}=\frac{S_{\text {branch }}}{\rho . l_{\text {branch }}}
$$

$\rho, l_{\text {branch }}$ and $S_{\text {branch }}$ are respectively branch material resistivity, length and section.

Because all nodes locations are chosen to be fixed, only branches sections can be changed and they will be defined as the optimization variables.

Moreover, the mass of a branch can be expressed as:

$$
M_{\text {branch }}=\mu_{\text {mat }} . l_{\text {branch }} . S_{\text {branch }}
$$

where $\mu_{m a t}$ is the material density. The total mass of the network is calculated as the sum of the mass of all branches. As it is not intended to increase the mass during the optimization, it is kept constant to its value $M_{0}$ before optimization. Finally, the optimization problem can be formulated as:

$$
\begin{gathered}
\text { min } \\
\text { subject to } \quad \sum_{l_{b} \leq S_{\text {branch }} \leq \theta_{2}}=u_{0}
\end{gathered}
$$

In this formulation, $\theta_{2}$ is maximized by minimizing $1 / \theta_{2}$. Branch sections are bounded between a lower value $l_{b}$ and an upper value $u_{b}$, where the lower value has to be positive. This formulation raises two issues. It requires the computation of the algebraic connectivity at each iteration of the optimization process. It may be time consuming. Furthermore, this optimization problem is not guaranteed to be convex, meaning that the global minimum may be hard to be reached.

\subsection{LMI-based formulation}

To avoid the calculation of eigenvalues, numerous optimization problems are transformed into Linear Matrix Inequality problems (LMI) based on positive semi-definite matrix properties. It is worth mentioning the Generalized Eigenvalue Problem that consists in minimizing the largest eigenvalue [7]. Moreover, LMI problems can be solved numerically very efficiently using interior-point methods.

The previous optimization problem is transformed into an alternative problem that consists in imposing a LMI constraint to the set of eigenvalues of the Laplacian matrix [26]. The constraint is defined by introducing a variable $\lambda$ and a matrix $Q$ with dimensions $n \times(n-1)$. The columns of $Q$ are the eigenvectors associated to the non-zero eigenvalues. The constraint is written as:

$$
Q^{t}(L+\lambda E) Q \succcurlyeq 0 \text {. }
$$

Thanks to the orthogonal properties of matrix $Q$, the inequality (28) means that all the eigenvalues, except the first one $\theta_{1}$ which is equal to zero, are higher than $-\lambda$. The minimization of $\lambda$ therefore leads to a maximization of the algebraic connectivity.

The LMI-based optimization formulation is finally written as:

$$
\begin{array}{cc}
\min & \lambda \\
\text { subject to } & Q^{t}(L+\lambda E) Q \succcurlyeq 0 \\
& \sum_{l_{b} \leq S_{\text {branch }} \leq u_{b} .} .
\end{array}
$$

The values of branch sections $S_{\text {branch }}$ control the optimization. Laplacian matrix is defined by Eq. (1). According to [7], the LMI constraint is convex. And the two supplementary constraints on mass equality and sections are 


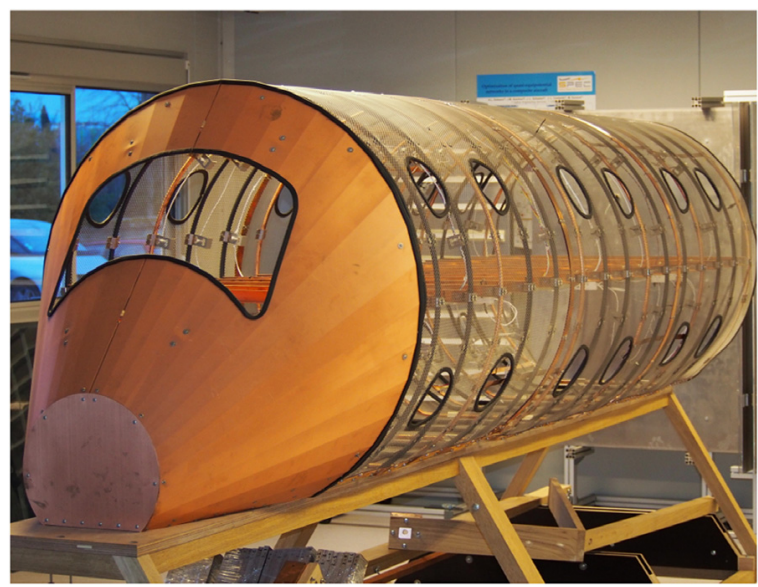

Fig. 4. DEMLAB mock-up at Safran Electrical \& Power's laboratory.

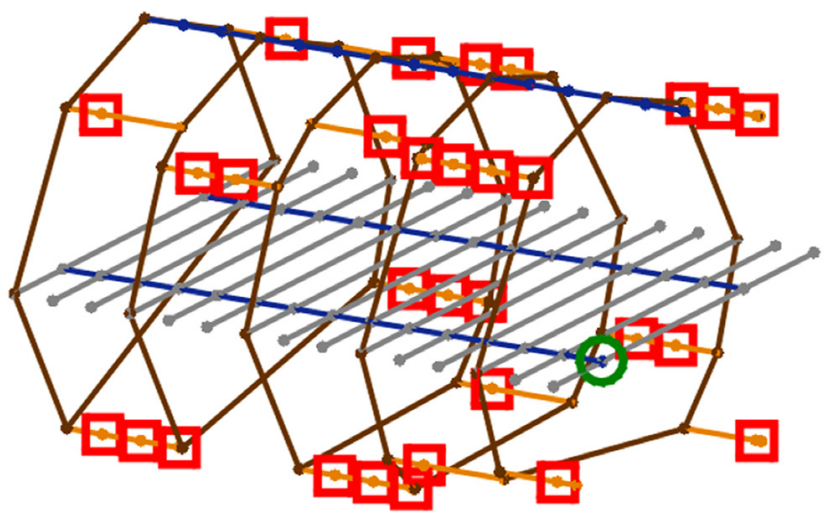

Fig. 5. DEMLAB's graph with source location (green circle) and possible load locations (red squares).

linear. Therefore, this formulation yields to a convex problem. This should make easier the solving because any local minimum will be a global minimum.

\section{Application to a current return network}

\subsection{Presentation of the network}

A reduced-scale mock-up of a current return network has been built by Safran Electrical \& Power. It is called DEMLAB and is shown in Fig. 4.

The mock-up is $3.6 \mathrm{~m}$ long and $1 \mathrm{~m}$ wide. The main conductive elements are the cabin floor, the frames, the raceways, the crossbeams and some copper strips. They are connected together to constitute a low impedance meshed network. It was previously used for validating some numerical solvers for low frequencies problems (grounding in normal conditions, lighting current carrying), reflectometry techniques and contact resistance measurements. One current source is located at one end of the network, and one load can be connected at 30 different nodes.

DEMLAB's graph is shown in Fig. 5. It has 244 nodes and 284 branches. All the branches are made of copper, and their sections are between 32 and $112 \mathrm{~mm}^{2}$. 


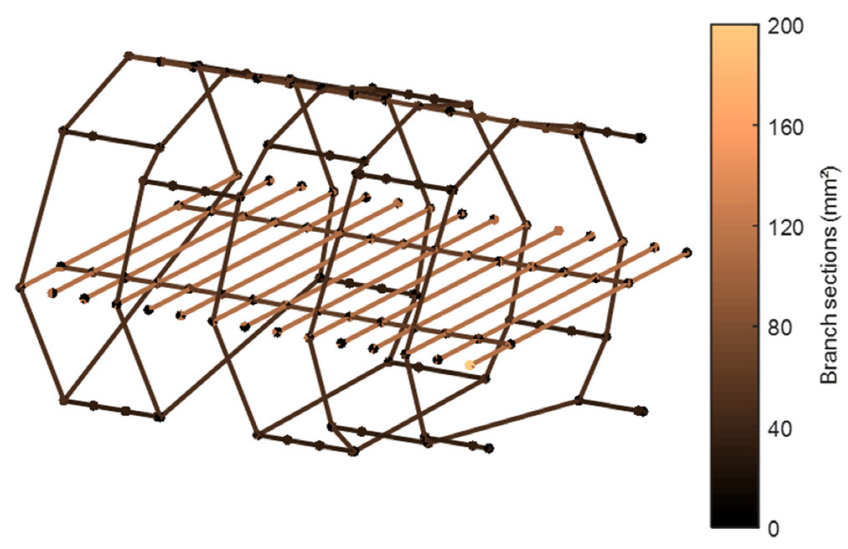

Fig. 6. Branch sections of DEMLAB before optimization.

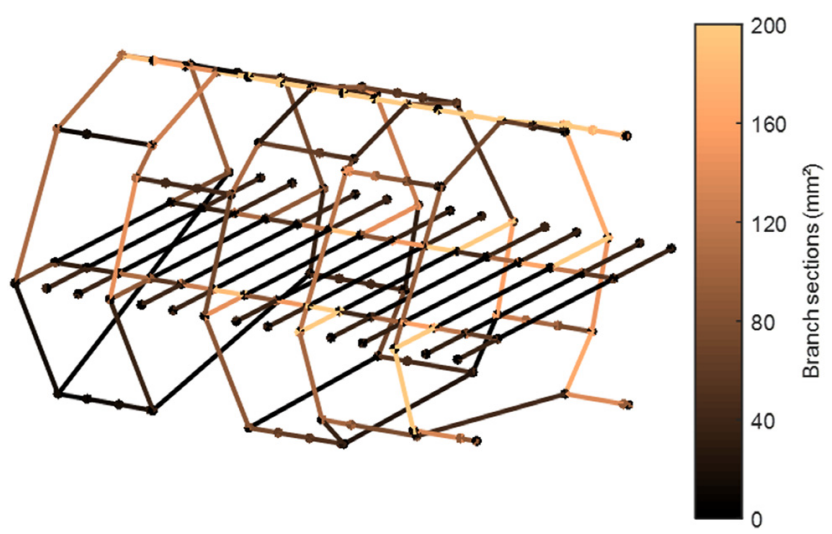

Fig. 7. Branch sections of DEMLAB after optimization.

\subsection{Optimization results}

The two formulations introduced in the previous section are now used for optimizing DEMLAB's network. Both of them have been implemented in Matlab. The first optimization is solved by the Fmincon function [8]. The second formulation is performed using the Dual-Scaling Semidefinite Programming method (DSDP) from the OPTimization Interface (OPTI) Toolbox [3]. In both cases, the solver uses the interior point method with gradient calculation performed by a finite differences approximation. In addition, the DSDP solver exploits the LMI properties in order to enhance the optimization performances.

During the optimization process, branch sections are allowed to vary between 2 and $200 \mathrm{~mm}^{2}$. The mass of the network is constant and fixed at its initial value of $28.8 \mathrm{~kg}$. The initial value of the algebraic connectivity $\theta_{2}$ is 71.7 .

After optimization, both formulations provide a higher value of the algebraic connectivity. It has been increased by 9.8\%, reaching the value of 78.7. The modifications of the branch sections are shown in Figs. 6 and 7. The sections of the transverse elements of the cabin floor have been reduced whereas the sections of some top conductive elements and frames have been increased. It results in an improvement of the global connectivity while the total mass of the network is kept constant.

The optimization performances are summarized in Table 1. The solving of the LMI-based formulation is faster than the explicit maximization. Although both optimizations have nearly the same number of iterations, the explicit maximization is 2.5 times longer. The main reason is the calculation of $\theta_{2}$ at each iteration. On the other hand, the LMI formulation exploits positive semi-definiteness of Laplacian matrix and calculations of eigenvalues are not needed. 
Table 1

Optimization performances.

\begin{tabular}{lll}
\hline & Explicit maximization & LMI-based formulation \\
\hline Number of iterations & 73 & 68 \\
Problem building time [s] & 1,3 & 9,3 \\
Resolution time [s] & 668,4 & 227,4 \\
Total time [s] & 669,7 & 236,7 \\
\hline
\end{tabular}

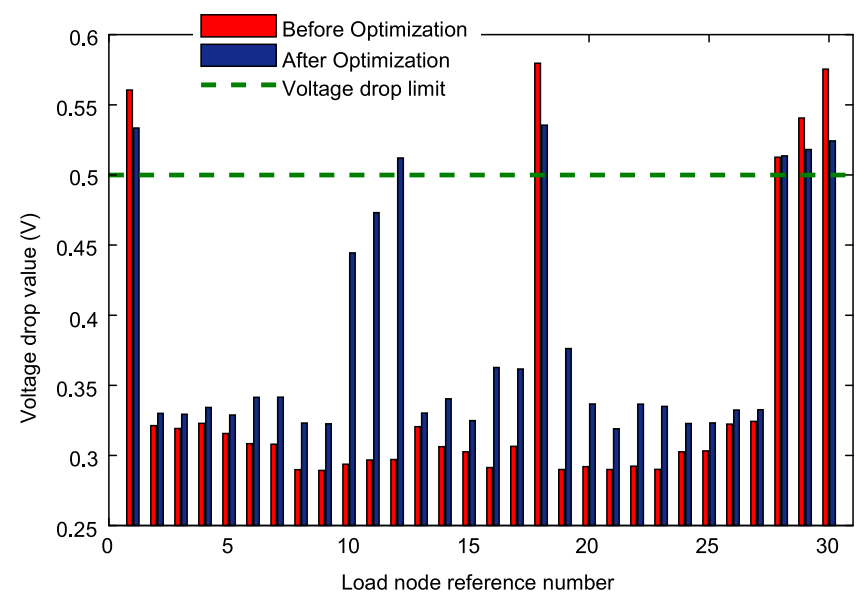

Fig. 8. Source-load voltage drops according to load location.

But the problem building is slower since it requires to calculate the eigenvectors of the Laplacian Matrix and to create the LMI constraint.

\subsection{Verifying voltage drop criteria}

As previously mentioned, the voltage drops at the loads connection nodes should not be higher than $0.5 \mathrm{~V}$ for a current return network operating in nominal conditions. As the proposed optimization approaches do not include voltage drop constraints, they have to be verified after processing the optimization.

For that purpose, a numerical circuit-oriented simulation of DEMLAB is performed. To simulate the nominal operating conditions, a 300 A DC current source is connected at node 212 (node circled in green in Fig. 5) and one single load is connected at any of the 30 possible load locations (nodes 1 to 30). Resulting voltage drops are presented in Fig. 8 according to load location.

Surprisingly, even if the network algebraic connectivity has been increased by the optimization, the network quality has been downgraded. Indeed, most of the voltage drops have been increased except for loads locations 1, 18, 29 and 30. To understand why such a surprising result has been obtained, a further look at path spectrum is required.

\subsection{Path spectrum analysis}

Firstly, the path between the source and node 18 is analysed. The spectrum decomposition of its DC resistance is expressed by (17). The values of the first 22 modal components of the decomposition are shown in Fig. 9. The other components are negligible.

It can be seen that, for this path, the most dominant mode is the second one. The optimization objective being the maximization of $\theta_{2}$, the DC connectivity has been increased by the optimization and therefore the voltage drop has been decreased as shown in Fig. 8. even still not below $0.5 \mathrm{~V}$.

On the other side, the spectrum of the path between the source and the node 12 is shown in Fig. 10. 


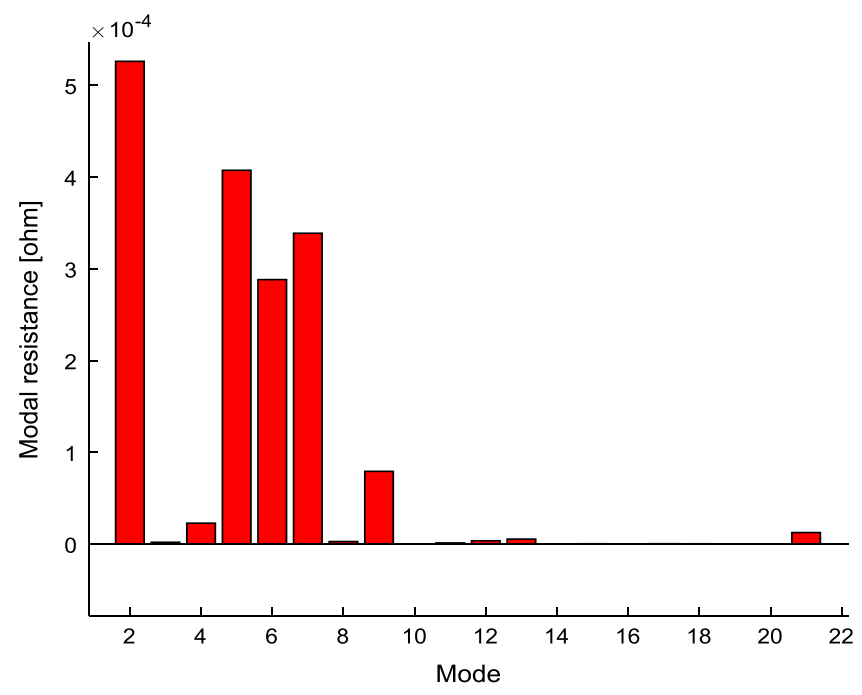

Fig. 9. Spectrum of path source-node 18 .

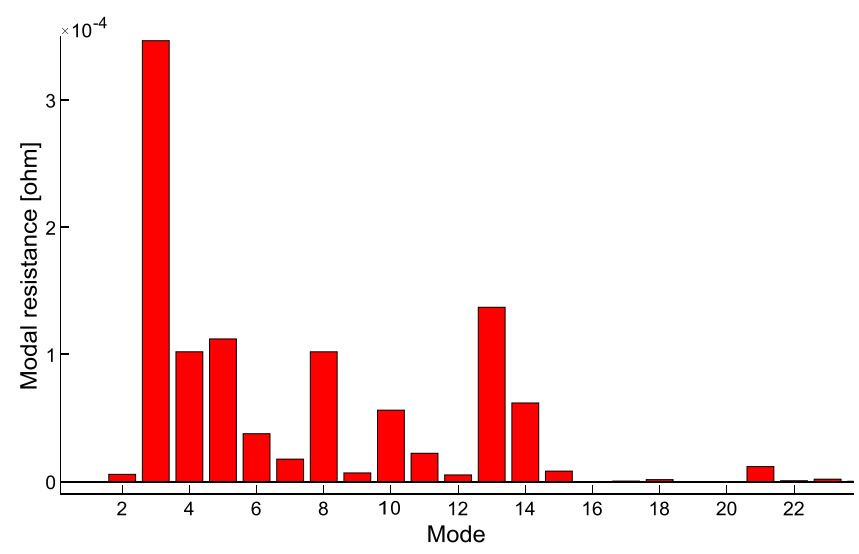

Fig. 10. Spectrum of path source-node 12 .

Table 2

Variation of some eigenvalues of path source-node12 after optimization.

\begin{tabular}{ll}
\hline Eigenvalue & Variation [\%] \\
\hline$\theta_{2}$ & 9.96 \\
$\theta_{3}$ & 2.45 \\
$\theta_{13}$ & -8.64 \\
$\theta_{5}$ & -8.86 \\
$\theta_{8}$ & -17.85 \\
$\theta_{4}$ & 1.87 \\
\hline
\end{tabular}

The five most dominant modes are the 3rd, 13th, 5th, 8th and 4th ones. Furthermore, the second mode is not influential at all.

Even if the optimization process has increased the algebraic connectivity, it has decreased at the same time the modes 5, 8 and 13 as shown in Table 2. As a result, the path resistance has been increased and therefore its voltage drop too. This is why most of the voltage drops have been deteriorated for almost all the loads location in spite of the higher algebraic connectivity. 
To conclude, enforcing $\theta_{2}$ may improve the connectivity of certain paths of the network. However, the voltage drops depend on all the eigenvalues, and increasing $\theta_{2}$ is not always sufficient to improve the overall voltage profile. For paths not depending dominantly on $\theta_{2}$, this optimization does not guarantee a better network performance. Our results show that it may even downgrade the voltage drops. A further optimization should be carried on to maximize all the dominant eigenvalues of the paths between electrical sources and loads. Another option is to optimize the DC resistance itself as a function of Laplacian eigenvalues [6].

\section{Conclusion}

In this paper, the DC connectivity of aircraft current return networks was analysed by using spectral graph analysis. Thanks to the multi-scale reading offered by the graph's spectrum, we show that a better connected network is achieved by increasing the second eigenvalue. It was also expected that a network with higher $\theta_{2}$ has lower voltage drops.

An LMI-based algorithm was presented to perform the algebraic connectivity optimization of a meshed graph by changing sections of all branches while keeping the total mass constant. The algorithm was tested with success for a representative aircraft return current network. On the other hand, it has been shown that not all the voltage drops were improved by optimization because they are not all ruled by the algebraic connectivity.

Despite of these limitations, the developed approach is promising because it offers to scientists and engineers an in-depth dissection of complexity of meshed electrical pathways used for ensuring current return network functions in more composite aircraft. In addition, it provides a pre-design tool based on spectral optimization. Some theoretical works have also proven that it could certainly be used with success to perform in the future dynamic analysis of complex electrical networks or assess their robustness to the removal of some branches [20].

\section{References}

[1] FAST Magazine, Vol. 53, Jan 2014. http://www.airbus.com/support/publications.

[2] B. Ayazifar, Graph Spectra and Modal Dynamics of Oscillatory Networks (Ph.D. thesis), Massachusetts Institute of Technology, 2002.

[3] S.J. Benson, Y. Ye, DSDP5: Software for semidefinite programming, ACM Trans. Math. Software (2005).

[4] S. Boccaletti, V. Latora, Y. Moreno, M. Chavez, D.-U. Hwang, Complex networks: Structure and dynamics, Phys. Rep. 424 (4-5) (2006) $175-308$.

[5] E. Bompard, E. Pons, D. Wu, Extended topological metrics for the analysis of power grid vulnerability, IEEE Syst. J. 6 (3) (2012) $481-487$.

[6] S. Boyd, Convex optimization of graph Laplacian eigenvalues, in: International Congress of Mathematicians, Vol. 3, 2006, pp. $1311-1319$.

[7] S. Boyd, L. El Ghaoui, E. Feron, V. Balakrishnan, Linear Matrix Inequalities in System and Control Theory, Society for Industrial and Applied Mathematics, 1994

[8] R.H. Byrd, J.C. Gilbert, J. Nocedal, A trust region method based on interior point techniques for nonlinear programming, Math. Program. 89 (1) (2000) 149-185.

[9] J.-G. Caputo, A. Knippel, E. Simo, Oscillations of networks: the role of soft nodes, J. Phys. A 46 (2013) 035101.

[10] F.R. Chung, Laplacians of graphs and Cheeger's inequalities, Comb. Paul Erdos Eighty 2 (157-172) (1996).

[11] E. Cotilla-Sanchez, P.D.H. Hines, C. Barrows, S. Blumsack, Comparing the topological and electrical structure of the north american electric power infrastructure, IEEE Syst. J. 6 (4) (2012) 616-626.

[12] Y. Dekel, J.R. Lee, et N. Linial, Eigenvectors of random graphs: Nodal domains, Random Structures Algorithms 39 (1) (2011) $39-58$.

[13] F. Dorfler, F. Bullo, Spectral analysis of synchronization in a lossless structure-preserving power network model, in: 2010 First IEEE International Conference on Smart Grid Communications, Gaithersburg, MD, 2010, pp. 179-184.

[14] V. Fioriti, M. Sforna, et G. D’Agostino, Spectral analysis of a real power network, Int. J. Crit. Infrastruct. 8 (4) (2012) 354-367.

[15] R.E. Gomory, T.C. Hu, Multi-terminal network flow, J. Soc. Ind. Appl. Math. 9 (4) (1961).

[16] G. Gutierrez, et al., On the design of aircraft electrical structure networks, IEEE Trans. Electromagn. Compat. 58 (2) (2016) $401-408$.

[17] N.C. Hien, N. Mithulananthan, R.C. Bansal, Location and sizing of distributed generation units for loadabilty enhancement in primary feeder, IEEE Syst. J. 7 (4) (2013) 797-806.

[18] P. Kundur, Power System Stability and Control, McGraw-Hill Professional, New-York, 1994.

[19] H. Liu, M. Cao, C.W. Wu, Coupling strength allocation for synchronization in complex networks using spectral graph theory, IEEE Trans. Circuits Syst. I. Regul. Pap. 61 (5) (2014) 1520-1530.

[20] M.W. Mahoney, Lecture Notes on Spectral Graph Methods, 2016 arXiv preprint arXiv:1608.04845.

[21] G. Marsh, Airbus takes on boeing with reinforced plastic A350 XWB, Reinforced Plast. 51 (11) (2007) 26-29.

[22] M.E.J. Newman, A measure of betweenness centrality based on random walks, Soc. Netw. 27 (1) (2005) 39-54.

[23] G.A. Pagani, M. Aiello, The power grid as a complex network: a survey, Physica A 392 (11) (2013) 2688-2700.

[24] N. Rubido, Energy Transmission and Synchronization in Complex Networks: Mathematical Principles (Thesis), Springer, 2016.

[25] S. Segarra, A. Ribeiro, Stability and continuity of centrality measures in weighted graphs, IEEE Trans. Signal Process. 64 (3) (2016) 543-555.

[26] S.Y. Shafi, M. Arcak, L. El Ghaoui, Designing node and edge weights of a graph to meet Laplacian eigenvalue constraints, in: 48th Annual Allerton Conference on Communication, Control, and Computing (Allerton), Allerton, IL, 2010, pp. 1016-1023. 